\title{
Single oral fixed-dose praziquantel-miltefosine nanocombination for effective control of experimental schistosomiasis mansoni
}

\author{
Maha M. Eissa ${ }^{1}$, Mervat Z. El-Azzouni ${ }^{1}$, Labiba K. El-Khordagui ${ }^{2}$ (C), Amany Abdel Bary ${ }^{3}$, Riham M. El-Moslemany ${ }^{2}$
} and Sara A. Abdel Salam ${ }^{1}$

\begin{abstract}
Background: The control of schistosomiasis has been centered to date on a single drug, praziquantel, with shortcomings including treatment failure, reinfection, and emergence of drug resistance. Drug repurposing, combination therapy or nanotechnology were explored to improve antischistosomal treatment. The aim of the present study was to utilize a novel combination of the three strategies to improve the therapeutic profile of praziquantel. This was based on a fixed-dose nanocombination of praziquantel and miltefosine, an antischistosomal repurposing candidate, co-loaded at reduced doses into lipid nanocapsules, for single dose oral therapy.
\end{abstract}

Methods: Two nanocombinations were prepared to provide $250 \mathrm{mg}$ praziquantel-20 mg miltefosine/ $\mathrm{kg}$ (higher fixed-dose) or $125 \mathrm{mg}$ praziquantel-10 mg miltefosine/kg (lower fixed-dose), respectively. Their antischistosomal efficacy in comparison with a non-treated control and their praziquantel or miltefosine singly loaded counterparts was assessed in murine schistosomiasis mansoni. A single oral dose of either formulation was administered on the initial day of infection, and on days 21 and 42 post-infection. Scanning electron microscopic, parasitological, and histopathological studies were used for assessment. Preclinical data were subjected to analysis of variance and Tukey's post-hoc test for pairwise comparisons.

Results: Lipid nanocapsules ( $58 \mathrm{~nm}$ ) showed high entrapment efficiency of both drugs (> 97\%). Compared to singly loaded praziquantel-lipid nanocapsules, the higher nanocombination dose showed a significant increase in antischistosomal efficacy in terms of statistically significant decrease in mean worm burden, particularly against invasive and juvenile worms, and amelioration of hepatic granulomas $(P \leq 0.05)$. In addition, scanning electron microscopy examination showed extensive dorsal tegumental damage with noticeable deposition of nanostructures.

Conclusions: The therapeutic profile of praziquantel could be improved by a novel multiple approach integrating drug repurposing, combination therapy and nanotechnology. Multistage activity and amelioration of liver pathology could be achieved by a new praziquantel-miltefosine fixed-dose nanocombination providing $250 \mathrm{mg}$ praziquantel-20 mg miltefosine/kg. To the best of our knowledge, this is the first report of a fixed-dose nano-based

*Correspondence: lakhalil@alexpharmacy.edu.eg; lakhalil@gmail.com

2 Department of Pharmaceutics, Faculty of Pharmacy, Alexandria University, Alexandria, Egypt

Full list of author information is available at the end of the article

c) The Author(s) 2020. This article is licensed under a Creative Commons Attribution 4.0 International License, which permits use, sharing, adaptation, distribution and reproduction in any medium or format, as long as you give appropriate credit to the original author(s) and the source, provide a link to the Creative Commons licence, and indicate if changes were made. The images or other third party material in this article are included in the article's Creative Commons licence, unless indicated otherwise in a credit line to the material. If material is not included in the article's Creative Commons licence and your intended use is not permitted by statutory regulation or exceeds the permitted use, you will need to obtain permission directly from the copyright holder. To view a copy of this licence, visit http://creativeco mmons.org/licenses/by/4.0/. The Creative Commons Public Domain Dedication waiver (http://creativecommons.org/publicdomain/ zero/1.0/) applies to the data made available in this article, unless otherwise stated in a credit line to the data. 
combinatorial therapy for schistosomiasis mansoni. Further studies are needed to document the nanocombination safety and explore its prophylactic activity and potential to hinder the onset of resistance to the drug components.

Keywords: Praziquantel, Miltefosine, Lipid Nanocapsules, Schistosoma mansoni, Nanocombination, Multistage activity, Tegumental targeting, Scanning electron microscopy

\section{Background}

Schistosomiasis is a debilitating parasitic disease affecting more than 200 million people over 78 tropical and subtropical countries, of which a significant number are preschool children $[1,2]$. The disease is caused by three main species of the genus Schistosoma having complex life-cycles that involve an intermediate snail host and a definitive human host. Schistosoma mansoni, the causative agent of intestinal schistosomiasis, is acquired by human contact with freshwater containing infectious cercariae. Egg deposition in the liver is associated with the formation of granulomas and subsequent serious complications [1]. In addition, both adult schistosomes and deposited eggs induce immunomodulatory effects that impair the host immune defences against other pathogens, such as human immunodeficiency and hepatitis $\mathrm{B} /$ hepatitis $\mathrm{C}$ viruses [3, 4].

No effective vaccine exists to date and chemopreventive therapy of schistosomiasis has relied for decades on praziquantel (PZQ), a low-cost well-tolerated drug with proven efficacy against the major species of schistosomiasis. According to the World Health Organization, PZQ is administered at the standard single oral dose of $40 \mathrm{mg} / \mathrm{kg}$ body weight in mass drug administration (MDA) programmes in endemic countries. Although such campaigns resulted in reduction of worm burden, incomplete cure attributed to ineffectiveness of PZQ against immature juvenile worms and reinfection have been reported [5, 6]. Moreover, drug resistance due to the widespread use of PZQ presents a serious threat to the gains achieved [7].

Taking these concerns into consideration and given the modern resources of drug development, synthesis of PZQ derivatives [8], discovery of alternative lead compounds $[9,10]$, natural products $[11,12]$ and repurposing of existing drugs $[13,14]$ are effective approaches for the introduction of new antischistosomal agents. Recently, we demonstrated that miltefosine (MFS), a membrane active alkylphosphocholine, is a promising repurposing candidate against schistosomiasis $[13,15]$. The drug showed multistage activity in five successive $20 \mathrm{mg} / \mathrm{kg} /$ day oral doses in mice which could be significantly enhanced by lipid nanoencapsulation, allowing for a $20 \mathrm{mg} / \mathrm{kg}$ single dose oral therapy $[16,17]$.

Nevertheless, building on the clinical and pharmacoeconomic merits of PZQ, more success can be achieved by overcoming the main limitations of PZQ therapy. Application of new strategies, such as nanotechnology and combinational therapy may extend the useful life of PZQ in more effective new formulations. In this respect, pharmaceutical nanotechnology may address challenges such as inadequate solubility, bioavailability, cellular delivery as well as non-specific biodistribution and rapid clearance of antiparasitic drugs $[18,19]$. Indeed, drug delivery systems including lipidbased nanocarriers such as liposomes [20] and solid lipid nanoparticles [21], niosomes [22] and silica nanoparticles [23] were shown to enhance the bioavailability and antischistosomal activity of PZQ. Recently, we demonstrated that entrapment of PZQ into lipid nanocapsules (LNCs) significantly enhanced its antischistosomal activity in a single oral reduced dose of $250 \mathrm{mg} /$ $\mathrm{kg}$ in mice [24]. LNCs are nanostructures with great potentials in drug delivery [25-27]. Owing to their relatively small and controllable size (20-100 nm), structural integrity in simulated gastrointestinal (GI) fluids and possible active transport across the intestinal epithelium, LNCs are highly promising as oral nanovectors $[28,29]$. In schistosomiasis treatment, oral LNCs also showed potential S. mansoni tegumental targeting [24].

Apart from nanotechnology, drug combination therapy aiming at synergies, resistance reduction and rejuvenation of old drugs, is another approach showing increasing benefits in the treatment of several diseases, particularly cancer [30, 31] and bacterial infections [32]. In experimental schistosomiasis, promising results have been reported for combinations involving PZQ and other drugs or biomolecules, aiming at multistage targeting, amelioration of infection-associated pathologies and resistance reduction [12, 14]. Despite evident advantages, combinations of free drugs may display variation in the pharmacokinetics and membrane transport among component drugs in addition to intricate dosing, resulting in inadequate outcomes [33]. Such limitations led to the emergence of an innovative combination therapy approach based on multi-drug delivery nanocarriers with increasing benefits in diverse diseases, notably cancer $[34,35]$. Although still in an early stage in the treatment of infectious diseases [36], the carrier-mediated multiple drug approach proved promising in the treatment of malaria $[37,38]$. 
To date, the research on nanocarrier-mediated drug delivery against schistosomiasis has focussed mainly on monotherapy [39]. Thus, the objective of the present study was to improve the therapeutic profile of PZQ by overcoming its shortcomings, mainly ineffectiveness against immature worms and inadequate liver protection. In addition, combination therapy could have the potential to overcome the problem of reinfection as well as offer protection of the component drugs from development of resistance. Therefore, PZQ was combined with MFS with proven activity against all developmental stages of the parasite and liver protective effects. To allow single dose oral administration, imperative in MDA campaigns, without the intricate dosing and varying outcomes of free drug combinations, PZQ and MFS were co-loaded in LNCs as a single oral fixed-dose LNC nanocombination. The antischistosomal efficacy of the nanocombination was assessed at two dosing levels of both drugs in comparison with singly loaded PZQ LNCs in experimental schistosomiasis mansoni in mice. Scanning electron microscopy (SEM), parasitological parameters, and histopathological examination were utilized for antischistosomal efficacy assessment against the invasive, juvenile, and adult developmental stages of the parasite.

\section{Methods}

\section{Materials}

The following chemicals were used in the study: PZQ (high purity, gift of the Egyptian International Pharmaceuticals Industries Company (EIPICO), Cairo, Egypt); MFS (98-102\%, Chem-Impex International, New York, USA); Labrafac ${ }^{\circledR}$ lipophile WL 1349 (Gattefossé SA, Saint-Priest, France); Kolliphor HS 15 (BASF, Ludwigshafen, Germany); Lipoid S100 (a soybean lecithin containing $94 \%$ of phosphatidylcholine, Lipoïd GMBH, Ludwigshafen, Germany); Oleic acid (OA, > 93\%, SigmaAldrich Co., St Louis, MO, USA); acetonitrile HPLC grade (Thermo Fisher Scientific, Waltham, MA, USA); and $\operatorname{Span}^{\circledR} 80$ (extra pure, LobaChemie for Laboratory Reagents and Fine Chemicals, Mumbai, India). All other chemicals were of analytical grade.

\section{Formulation and characterization of lipid nanocapsules}

LNCs were formulated with oleic acid and Span 80 and prepared by the phase inversion method [24, 27]. In brief, Kolliphor ${ }^{\circledR}$ HS 15, Labrafac lipophile WL 1349 and deionized water containing $\mathrm{NaCl}(0.88 \% \mathrm{w} / \mathrm{w}$ of the final dispersion) were weighed and mixed using a magnetic stirrer in the ratio 5:6:9. Span $80(2 \% \mathrm{w} / \mathrm{w})$ and oleic acid $(6 \% \mathrm{w} / \mathrm{w})$ were added to the primary mixture which was subjected to three progressive heating and cooling cycles between $45-75^{\circ} \mathrm{C}$ at $4{ }^{\circ} \mathrm{C} / \mathrm{min}$. An irreversible shock was induced by a two-fold dilution with cold deionized water $\left(0-2{ }^{\circ} \mathrm{C}\right)$ added to the formed $\mathrm{o} / \mathrm{w}$ emulsion at a temperature $1-3{ }^{\circ} \mathrm{C}$ from the beginning of the phase inversion zone. This was followed by slow magnetic stirring at room temperature for $5 \mathrm{~min}$. For the preparation of drug loaded LNCs, PZQ was added to the primary mixture of ingredients at concentrations 25 or $12.5 \mathrm{mg} / \mathrm{ml}$, whereas MFS was added just before quenching at a concentration of 2 or $1 \mathrm{mg} / \mathrm{ml}$ of the final dispersion. The procedure was used to prepare a higher fixed-dose combination containing $25 \mathrm{mg}$ PZQ and $2 \mathrm{mg} \mathrm{MFS} / \mathrm{ml}$ of dispersion and a lower fixed-dose combination containing $12.5 \mathrm{mg}$ PZQ and $1 \mathrm{mg} \mathrm{MFS} / \mathrm{ml}$ of dispersion and their corresponding singly loaded counterparts, PZQ $25 \mathrm{mg} / \mathrm{ml}$, MFS $2 \mathrm{mg} /$ $\mathrm{ml}$, PZQ $12.5 \mathrm{mg} / \mathrm{ml}$ and MFS $1 \mathrm{mg} / \mathrm{ml}$. The fixed-dose combination LNC dispersions were used in calculated volumes to provide a dose of $250 \mathrm{mg} / \mathrm{kg}$ PZQ-20 mg/kg MFS and $125 \mathrm{mg} / \mathrm{kg}$ PZQ- $10 \mathrm{mg} / \mathrm{kg}$ MFS respectively in the antischistosomal study in mice.

LNC formulations were characterized for morphology, colloidal properties, and drug entrapment efficiency (EE\%). The morphology of LNCs was examined by transmission electron microscopy (TEM) using JEOL, JEM-100 CX electron microscope, Tokyo, Japan. Before analysis, the LNC dispersion was treated with $2 \% \mathrm{w} / \mathrm{v}$ uranyl acetate solution as a negative stain and sprayed onto copper grids. Shots were taken at $\times 5000$ at $80 \mathrm{kV}$. The average particle size, polydispersity index (PdI) and zeta potential (ZP) were measured by photon correlation spectroscopy (PCS) at a fixed angle $173^{\circ}$ using a $4 \mathrm{~mW}$ $\mathrm{He}-\mathrm{Ne}$ laser at $25{ }^{\circ} \mathrm{C}$. The EE\% was obtained by determining the concentration of free (unentrapped) PZQ and MFS in the ultrafiltrate after separation of LNCs using an ultrafiltration/centrifugation technique. The concentration of unentrapped PZQ in the ultrafiltrate was determined by HPLC-UV as previously reported [24]. MFS concentration was measured by a modified spectrophotometric assay originally reported for quaternary ammonium compounds and validated for MFS quantitation [16].

\section{Antischistosomal efficacy in mice Schistosoma mansoni infection of animals}

The life-cycle of S. mansoni was maintained in the Medical Parasitology Department, Faculty of Medicine, Alexandria University by serial passages in laboratorybred Biomphalaria alexandrina snails and Swiss albino mice [40]. A total of 128 mice, 6-8 weeks-old, weighing 20-30 g each, were obtained from the animal house of the Medical Parasitology Department, Faculty of Medicine, Alexandria University. Mice were housed under specific pathogen-free barrier conditions. Each mouse was infected with $100 \pm 10$ freshly shed cercariae using 
the paddling technique [41]. All mice were subjected to infection.

\section{Animal groups}

A total of 128 mice were allocated to a non-treated control group (NT) including 8 mice and 2 experimental groups, Group I and Group II. Group I $(n=60)$ was subdivided into 3 subgroups as follows: Subgroup Ia, PZQ $250 \mathrm{mg} / \mathrm{kg}(n=20)$; Subgroup Ib, MFS $20 \mathrm{mg} / \mathrm{kg}$ $(n=20)$; and Subgroup Iab, PZQ $250 \mathrm{mg}$-MFS $20 \mathrm{mg} /$ $\mathrm{kg}(n=20)$. Group II $(n=60)$ was subdivided into 3 subgroups as follows: Subgroup IIa, PZQ $125 \mathrm{mg} / \mathrm{kg}$ $(n=20)$; Subgroup IIb, MFS $10 \mathrm{mg} / \mathrm{kg}(n=20)$; and Subgroup IIab, PZQ 125-MFS $10 \mathrm{mg} / \mathrm{kg}(n=20)$.

Mice in all treated subgroups (Ia, Ib and Iab, IIa, IIb and IIab) were administered a calculated volume of the LNCs dispersion corresponding to the required dose of the PZQ-MFS fixed-dose combinations or the corresponding singly loaded LNCs by gastric gavage. Twenty mice in these subgroups were further subdivided into 3 subgroups (1,2 and 3 ) which were given a single oral dose of the drug(s) nanoformulations at 3 different dates (on the initial day of infection, and days 21 and 42 postinfection (dpi)) corresponding to the three stages of $S$. mansoni life-cycle (invasive, juvenile and adult stages), respectively [17]. The number of mice/group treated on the initial day of infection and 21 dpi (against invasive and immature stages) was 6 whereas, it was 8 for all other subgroups treated on day 42 post-infection (p.i.) (against the adult stage). Two infected mice treated on day $42 \mathrm{p}$.i. were sacrificed $24 \mathrm{~h}$ after administration of the nanoformulations to collect the adult worms for morphological examination using SEM (Jeol JSM- IT200, Jeol, Tokyo, Japan). The remaining mice of all subgroups were sacrificed on day 49 p.i. The therapeutic efficacy of the two fixed-dose nanocombinations in comparison with the corresponding singly loaded control LNCs and the non-treated control was assessed by determination of the percentage reduction in total worm burden, the size of hepatic granulomas, histopathological changes in liver parenchyma and examination of the morphology of recovered worms by SEM.

\section{Antischistosomal activity assessment Estimation of adult worm burden}

On day 49 p.i., adult worms were recovered from the hepatic and mesenteric vessels from mice in all study groups using the perfusion technique [41].

Morphological examination by scanning electron microscopy Adult worms were recovered $24 \mathrm{~h}$ post-administration of nanoformulations from two infected mice from all subgroups treated on day 42 p.i. against the adult stage for scanning electron microscopic (SEM) imaging. Worms were fixed in cold $2.5 \%$ buffered glutaraldehyde phosphate ( $\mathrm{pH} 7.4$ ), washed, dehydrated through an ascending series of ethanol, and left to dry in air. After drying, they were embedded in epoxy resin, mounted on aluminium stubs and coated with $20 \mathrm{~nm}$ gold particles in an ionsputtering apparatus. Adult male worms were examined under SEM and photographed [42].

\section{Histopathological examination}

Samples of the liver of mice of all study groups were fixed in $10 \%$ neutral buffered formalin. Parrafin-embedded histological sections ( $5 \mu \mathrm{m}$-thick) were stained with hematoxylin and eosin (H\&E) [43]. Pathological changes in the hepatic parenchyma were observed and the mean size of granulomas was determined. Only granulomas containing one central clearly identifiable egg were selected. Their diameters were measured with a light microscope, equipped with an ocular micrometer under low power magnification $(100 \times)$. In each mouse, the mean diameter was calculated from 10 granulomata. Then, the mean granulomata size was estimated for each [24].

\section{Statistical analysis}

Data were analysed using IBM SPSS software package version 20.0 (IBM Corp, Armonk, NY, USA). Kolmogorov-Smirnov test was used to verify the normality of distribution. Quantitative data were described using mean and standard deviation. Significance of the obtained results was judged at the $5 \%$ level. For normally distributed quantitative variables, ANOVA F-test was used to compare between more than two groups and Tukey's post-hoc test was used for pairwise comparisons. The percentage reduction (\% R) of the adult worm load as well as the granuloma size were calculated as follows:

$$
\text { Percentage reduction }(\% \mathrm{R})=\frac{\mathrm{N}-n}{\mathrm{~N}} \times 100
$$

where $\mathrm{N}$ is the mean number of worms or the mean granuloma size in the infected non-treated group and $n$ is the mean number of worms or the mean granuloma size in the infected treated subgroups.

\section{Results}

\section{Characterization of lipid nanocapsules}

PZQ-MFS combination LNCs were prepared and characterized for size, PdI, ZP and EE\% as reported earlier for PZQ LNCs [24]. TEM imaging of LNCs (Fig. 1) showed almost spherical nanostructures, homogenously distributed, and not aggregated. Characterization data verified small size and monodispersity (PdI not exceeding 0.05). Blank LNCs had a mean diameter of $52.2 \pm 0.42 \mathrm{~nm}$ with a ZP of $-6.5 \pm 0.4 \mathrm{mV}$. Drug loading into LNCs slightly 
but significantly affected their size and ZP $(57.6 \pm 0.15$ $\mathrm{nm}$ and $-7.9 \pm 0.4 \mathrm{mV}$, respectively) as indicated by the t-test $\left(t_{(4)}=20.972, P<0.0001 ; t_{(4)}=4.287, P=0.0128\right.$, respectively). The EE\% calculated based on drug content exceeded $97 \%$ for both PZQ and MFS.

\section{Antischistosomal activity in S. mansoni-infected mice Assessment of adult worm burden}

Data for worm burden assessment in the study subgroups are shown in Fig. 2. There was a statistically significant difference between the different studied subgroups against invasive $\left(F_{(6,35)}=124.405\right.$, $P<0.001)$, juvenile $\left(F_{(6,35)}=185.422, P<0.001\right)$ and adult stages $\left(F_{(6,35)}=197.693, P<0.001\right)$. Oral administration of the two fixed-dose nanocombinations, $250 \mathrm{mg}$ PZQ-20 mg MFS/ $/ \mathrm{kg}$ (subgroups Iab 1, 2 and 3) and $125 \mathrm{mg}$ PZQ-10 mg MFS/kg (subgroups IIab 1, 2 and 3) to infected mice given against invasive, juvenile and adult worms resulted in a statistically significant reduction in the mean adult worm burden of $(6.17 \pm 1.47,3.0 \pm 0.89$ and $2.33 \pm 1.03)$ and $(15.50 \pm 1.87,21.0 \pm 3.90$ and $13.0 \pm 3.90)$ for the two subgroups, respectively, compared to a mean of $38.83 \pm 1.17$ for the infected non-treated control subgroup $(P<0.001)$. On comparing the two different doses of PZQ-MFS LNCs (subgroup Iab and subgroup IIab) together, a statistically significant reduction in the mean worm burden was observed in favour of the higher nanocombination dose given against the three different developmental stages (subgroups Iab 1, 2 and 3) $(P<0.001)$. There was also a statistically significant reduction in the mean adult worm burden of mice treated with a higher dose of PZQ-MFS LNCs (subgroup Iab) compared with PZQ-LNCs monotherapy given against invasive and juvenile stages (subgroups Ia 1 and 2) $(11.0 \pm 2.61$ and $29.33 \pm 2.50$, respectively $)$ $(P=0.044 ; P<0.001$, respectively).

Statistical analysis of worm burden data of the two fixed-doses PZQ-MFS LNCs treated subgroups against the different developmental stages of $S$. mansoni worms compared to the infected non-treated control, corresponding monotherapy controls as well as to each other are shown in Fig. 2.

\section{Morphological properties by SEM}

Results of the SEM examination are shown in Fig. 3. SEM images of normal $S$. mansoni male worms recovered from infected non-treated mice (NT) (Fig. 3a), showed round to oval oral and ventral suckers (Fig. 3b) with apically directed spines (Fig. 3c). The dorsolateral tegumental surface of the mid-body showed crab-like uniformly distributed tubercles that had sharp visible intact spines and sensory papillae (Fig. 3d). The tegument between

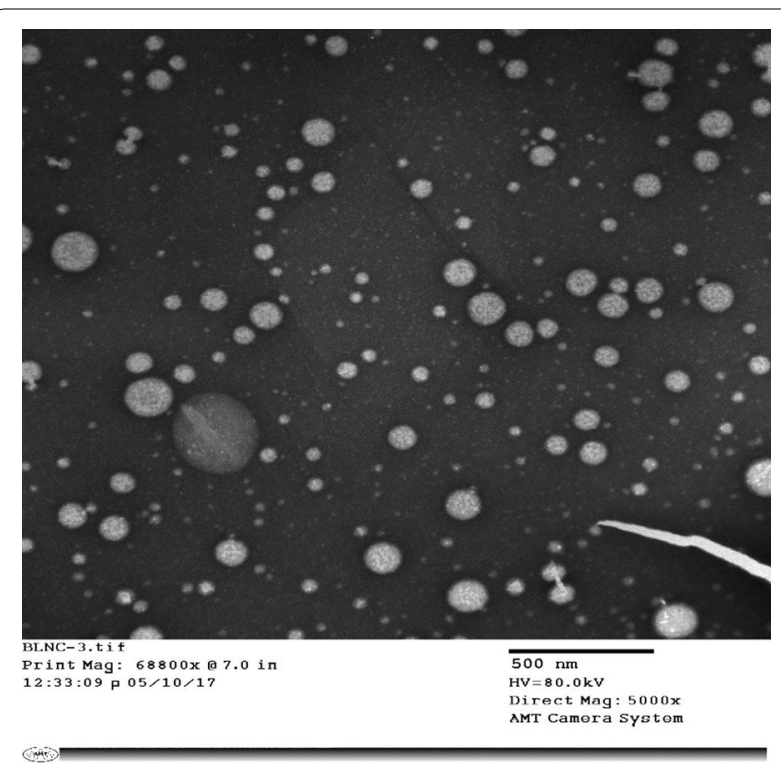

Fig. 1 TEM image of praziquantel-miltefosine combination lipid nanocapsules

the tubercles showed ridges (Fig. 3e). On the other hand, male worms recovered from $S$. mansoni-infected mice treated with the higher dose PZQ-MFS nanocombination (PZQ $250 \mathrm{mg}$-MFS $20 \mathrm{mg} / \mathrm{kg}$, Subgroup Iab) showed deformity in the whole body (Fig. 3f) as well as in the oral and ventral suckers (Fig. 3g), with blunt, short and loose spines (Fig. 3h). There was extensive dorsal tegumental damage in the form of peeling of tubercles and spine disfigurement with appearance of subtegumental tissues (Fig. 3i, j). Marked deposition of nanostructures of the size of LNCs on the damaged tegument (Fig. 3k) and on the loose disfigured spines (Fig. 3l) was observed. The tegumental changes and sucker deformity observed in infected mice treated with either PZQ $250 \mathrm{mg} / \mathrm{kg}$ or MFS $20 \mathrm{mg} / \mathrm{kg}$ monotherapy (control Subgroups Ia and Ib, respectively) were almost similar. Comparable but milder morphological changes were also observed in worms recovered from infected mice treated with the lower dose PZQ-MFS LNCs combination PZQ 125 mg-MFS $10 \mathrm{mg} /$ $\mathrm{kg}$ (Subgroup IIab) and the corresponding singly loaded LNCs (control Subgroups IIa and IIb).

\section{Assessment of histopathological changes in the liver}

As shown in Fig. 4, the histopathological changes detected in H\&E-stained liver sections of infected nontreated mice showed preserved hepatic architecture associated with granulomatous reaction located perivascularly and intraparenchymally (Fig. 4a). Most of the detected granulomas were active and formed mainly of eosinophils, lymphocytes and histocytes encircling laid 


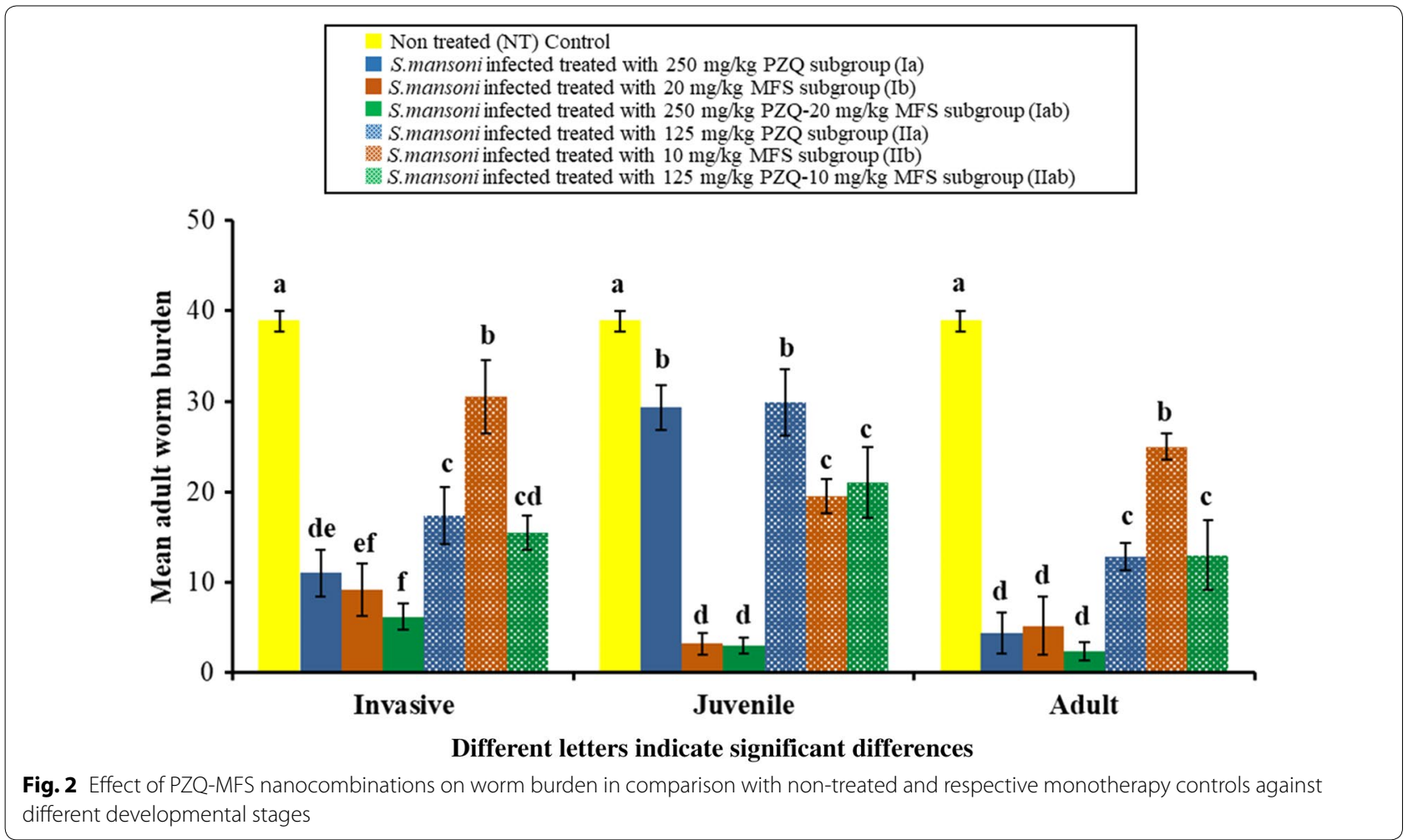

S. mansoni eggs (Fig. 4b). There was deposition of schistosomal pigments (Fig. 4c), Kupffer cells hyperplasia (Fig. 4d), and fatty changes in hepatocytes (Fig. 4e).

On the other hand, pronounced amelioration of $S$. mansoni-associated hepatic pathology was observed in all developmental stages in infected mice treated with the higher nanocombination dose (subgroup Iab 1, 2 and 3), manifested as scanty small healing granulomas formed of minimal inflammatory cellular infiltration and surrounded by concentric collagenous fibrous tissue (Fig. 4f). Marked improvement of hepatic pathology was also observed in all infected mice treated with singly loaded control LNCs at the higher dosing level (Subgroups Ia 1, 2 and 3 and Ib 1, 2 and 3). On the other hand, only minimal improvement in infection-associated hepatic pathological changes was observed in all infected mice treated with the lower nanocombination dose (Subgroups IIab 1, 2 and 3) as well as their corresponding singly loaded control LNCs (Subgroups IIa 1, 2 and 3 and IIb 1, 2 and 3).

Data for hepatic granuloma size in the study subgroups are shown in Fig. 5. There was a statistically significant difference between the different studied subgroups against invasive $\left(F_{(6,35)}=21.877, P<0.001\right)$, juvenile $\left(F_{(6}\right.$, $\left.{ }_{35)}=59.525, P<0.001\right)$ and adult stages $\left(F_{(6,35)}=73.989\right.$, $P<0.001)$. Oral administration of the higher dose PZQMFS nanocombination (PZQ $250 \mathrm{mg}-\mathrm{MFS} 20 \mathrm{mg} / \mathrm{kg}$, subgroups Iab 1, 2 and 3) to infected mice given against invasive, juvenile and adult worms resulted in a statistically significant reduction in the mean granuloma size $(214.6 \pm 18.47 \mu \mathrm{m}, 209.6 \pm 21.41 \mu \mathrm{m}$ and $153.5 \pm 12.0$ $\mu \mathrm{m}$, respectively) compared to a mean of $412.0 \pm 37.83$ $\mu \mathrm{m}$ for the infected non-treated control subgroup $(P<0.001)$. While the lower dose PZQ-MFS nanocombination (125 mg PZQ-10 mg MFS/kg, subgroups IIab 1, 2 and 3 ) given to infected mice against the three developmental stages induced a statistically significant reduction in the mean granuloma size $(314.5 \pm 44.01 \mu \mathrm{m}$, $385.4 \pm 27.20 \mu \mathrm{m}$ and $349.6 \pm 21.26 \mu \mathrm{m}$, respectively) compared to the infected non-treated control subgroup ( $P=0.001 ; P=0.555 ; P=0.003$, respectively). On comparing the two different doses of PZQ-MFS LNCs (subgroup Iab and subgroup IIab) together, a statistically significant reduction in the mean granuloma size was observed in favour of the higher nanocombination dose given against the three different developmental stages (subgroups Iab 1,2 and 3$)(P=0.001 ; P<0.001$; $P<0.001$, respectively). There was also a statistically significant reduction in the mean granuloma size of mice treated with higher dose of PZQ-MFS LNCs (subgroup Iab) compared to PZQ-LNCs monotherapy given against juvenile and adult stages (subgroups Ia 2 and 3) $(287.5 \pm 34.46 \mu \mathrm{m}$ and $230.6 \pm 7.02 \mu \mathrm{m}$, respectively) $(P<0.001)$. 

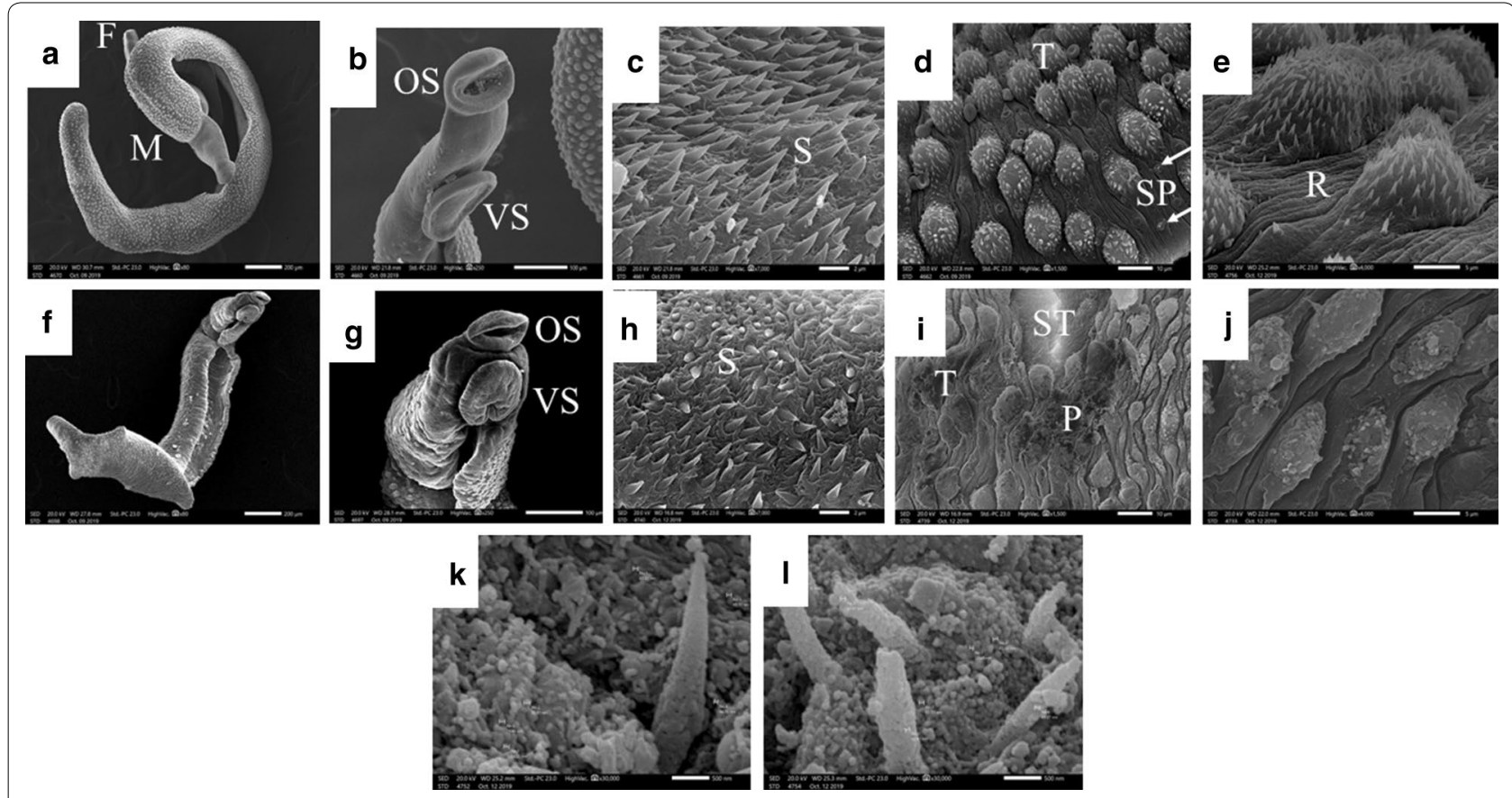

Fig. 3 Scanning electron micrographs of S. mansoni male worms recovered from the hepatic and mesenteric veins of infected non-treated mice (a-e) and infected mice treated with the higher PZQ-MFS nanocombination dose (PZQ 250 mg-MFS 20 mg/kg, subgroup lab) (f-I). a Normal male worm in copula with female $(\times 80)$. b Normal oral and ventral suckers $(\times 250)$ with apically directed spines $(\times 7000)(\mathbf{c})$. d Normal dorsolateral tegument surface of the mid body showing crablike uniformly distributed tubercles with sharp visible intact spines and sensory papillae $(x 1500)$. $\mathbf{e}$ Normal tegumental ridges between the tubercles $(\times 4000)$. Adult males recovered from S. mansoni-infected mice treated with the higher fixed-dose nanocombination (subgroup lab) $(\mathbf{f}-\mathbf{I})$. f Deformed whole body $(\times 80)$. $\mathbf{g}$ Deformed oral and ventral suckers $(\times 250)$. $\mathbf{h}$ Blunt, short and loose spines ( $\times 7000)$. i Extensive dorsal tegumental damage in the form of peeling of tubercles, spine disfigurement, vesicle formation and exposure of subtegumental tissue $(\times 1500)$. $\mathbf{j}$ Higher magnification of tegumental changes $(\times 4000)$. $\mathbf{k}$, I LNCs deposited on the damaged subtegumental tissue and the loose disfigured spines (×30000). Abbreviations: F, female; M, male; OS, oral sucker; VS, ventral sucker; S, spine; T, tubercle; SP, sensory papillae; $R$, ridge; $S T$, subtegumental tissue; $P$, peeling of the tubercles

Statistical analysis of granulomas size of the two fixeddoses PZQ-MFS LNCs treated subgroups against the different developmental stages of S. mansoni worms compared to infected non-treated control, corresponding monotherapy controls as well as to each other are shown in Fig. 5.

\section{Discussion}

We aimed at improving the antischistosomal therapeutic profile of PZQ in MDA programmes by enhancing multistage activity against the different developmental stages of the parasite, alleviating schistosomiasis-induced pathology and potentially reducing drug resistance while keeping dosage at a relatively low single oral dose. This cannot be achieved unless multiple strategies are exploited. In this study, an innovative approach based on the integration of the documented benefits of three strategies, namely drug repurposing, combination of multitarget drugs and nanotechnology, involved incorporation of PZQ and MFS in a nanocombination intended for single dose oral therapy of schistosomiasis mansoni.
As the key combination drug, PZQ exerts a direct antischistosomal effect on adult worms by underpinning the parasite $\mathrm{Ca}^{2+}$ homeostasis resulting in spastic paralysis and rapid vacuolization of the worm surface [44]. Recently, activation of a schistosome transient receptor potential channel by PZQ was shown to support the pharmacological profile of PZQ [45]. To overcome the known shortcomings of PZQ therapy, mainly ineffectiveness against immature worms and inability to ameliorate disease-associated pathology, MFS was included in the nanocombination. Selection of MFS relied on its current clinical use as the only approved oral drug for the treatment of visceral leishmaniasis [46] as well as earlier evidence of multistage antischistosomal activity of oral MFS in mice [13]. Although the antischistosomal mechanism of action of MFS is not well established yet, antiparasitic and anticancer effects of MFS appear to be related to its high affinity for plasma membrane $[47,48]$. As a membrane active zwitterionic alkylphospholipid, MFS causes dramatic increases in membrane dynamics by interacting with the protein component and inhibiting phospholipid 

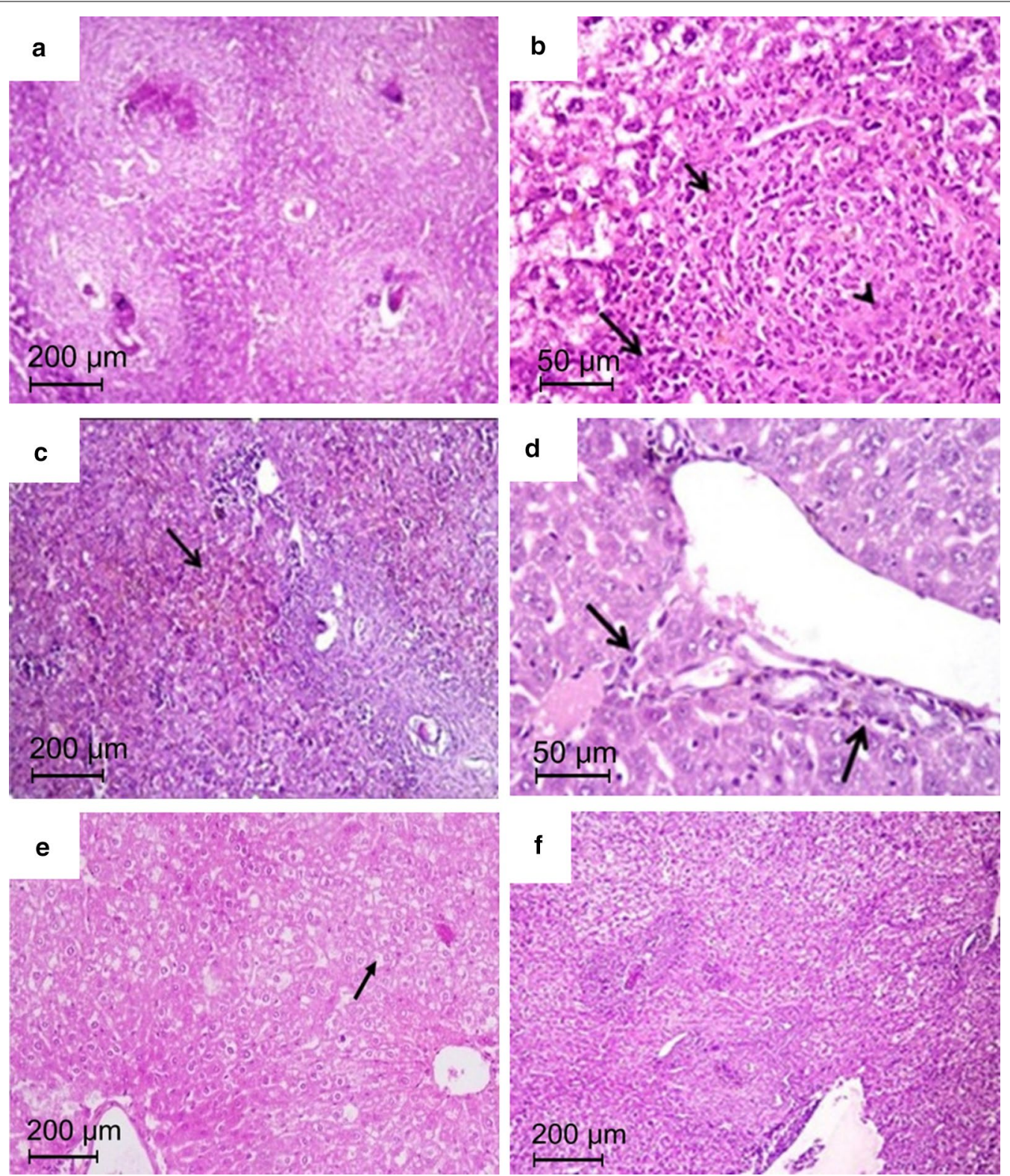

Fig. 4 H\&E-stained liver sections of S. mansoni-infected non-treated mice. a Preserved hepatic architecture and multiple numerous closely packed necrotic and exudative-productive granulomas ( $\times 100)$. b A granulomatous reaction formed of inflammatory cells mainly eosinophils (short arrows), neutrophils (long arrows) and histiocytes (arrowheads) (×400). c Brownish schistosomal pigment (arrow) (× 100). d Kupper cell hyperplasia (arrows) ( $\times 400)$. e Fatty change of hepatocytes (arrow) $(\times 100)$. $\mathbf{f}$ Liver section of $S$. mansoni-infected mice treated with the higher dose fixed combination (Subgroup lab) showing small-sized healing granulomas $(\times 100)$

turnover and lipid-dependent cell signaling pathways, leading to apoptosis. Of importance to schistosomiasis, MFS impedes the biosynthesis of sphingomyelin, a schistosome membrane phospholipid which hides the surface membrane proteins from the host immune system [49]. Evident damage to the Schistosoma tegument with exposure of their surface antigens was reported following treatment with MFS $[13,16,50]$.
Selection of LNCs as nanocarrier for the oral administration of the PZQ-MFS combination was based primarily on its recognized merits as lipid-based nanocarrier for oral drug delivery with modifiable composition. For example, LNCs were demonstrated to enhance the activity of MFS against $S$. mansoni compared to the free drug (67.51 vs $8.13 \%$ reduction in worm burden by MFS LNCs and free MFS respectively) with further enhancement 


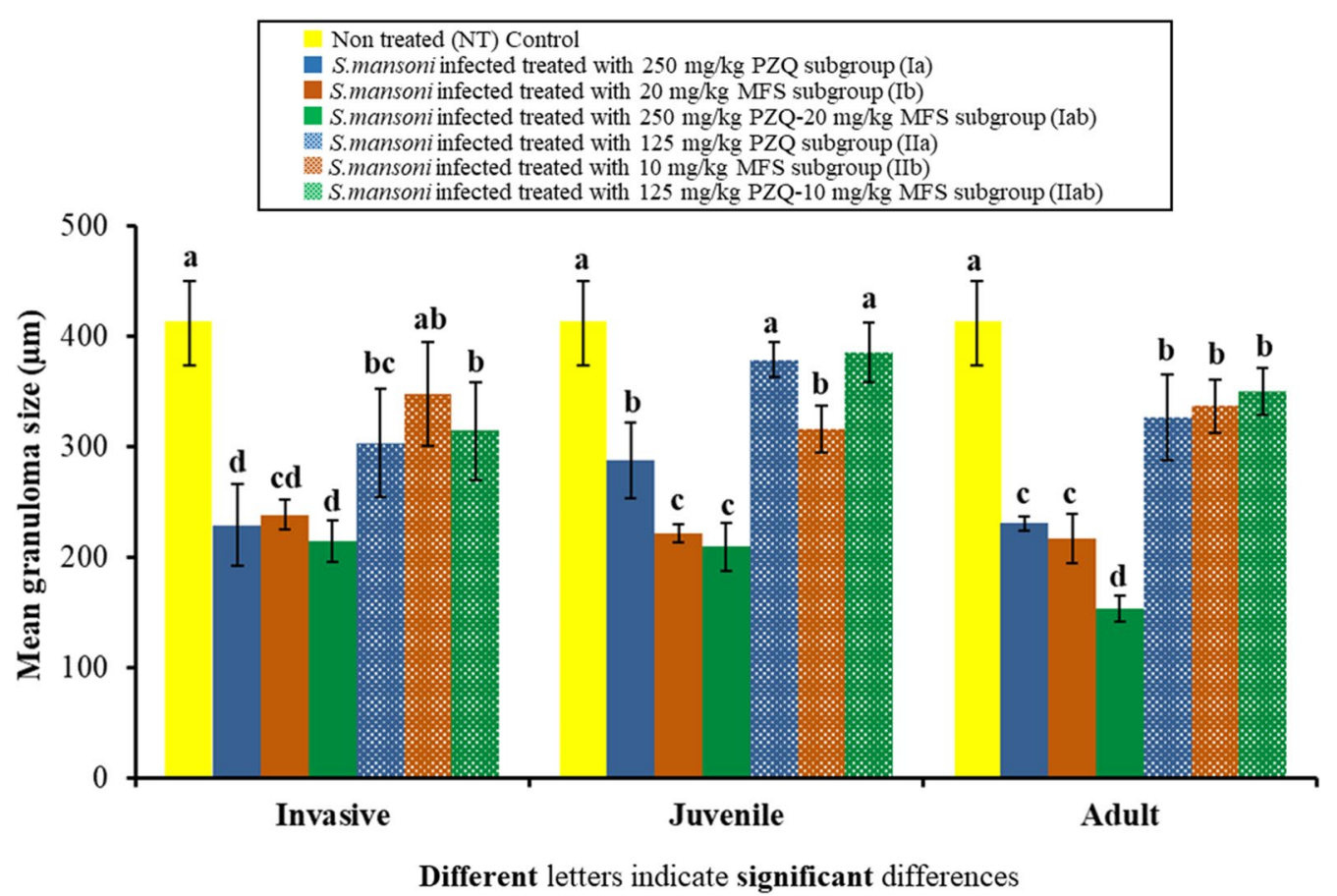

Fig. 5 Effect of PZQ-MFS nanocombinations on hepatic granuloma size in comparison with non-treated and monotherapy controls against different developmental stages

(88.46\% reduction in worm burden) upon modification of LNCs with oleic acid as membrane permeabilizer [16]. Owing to their amphiphilic nature, MFS molecules are possibly intercalated within the tensioactive shell of LNCs, enhancing their structural integrity and allowing for potential translocation across the intestinal membrane. A hypothesized structure of MFS-LNCs has been reported earlier [16]. LNCs modified with oleic acid and MFS as formulation additives also enhanced the activity of PZQ against experimental schistosomiasis mansoni [24]. In both MFS and PZQ systems, LNCs allowed marked sustained release of the active drugs for at least $24 \mathrm{~h}$.

Analysis of data obtained in the present study indicated that the activity of PZQ LNCs $(250 \mathrm{mg} / \mathrm{kg})$ against schistosomiasis mansoni was in the order of adult > invasive > juvenile while that of MFS LNCs $(20 \mathrm{mg} / \mathrm{kg})$ was in the order of juvenile $>$ adult $>$ invasive corroborating literature data for both drugs in the free form $[13,51,52]$ and nanoencapsulated form [16, 17, 24]. The nanocombination of MFS with PZQ in the same doses (PZQ 250 $\mathrm{mg} / \mathrm{kg}$-MFS $20 \mathrm{mg} / \mathrm{kg}$ ) resulted in high activity against prepatent and patent infection implying multistage activity, a main objective of the present study.

Reduction in mean adult worm burden (Fig. 2), the amelioration of hepatic pathology (Fig. 4) in addition to liver granuloma size (Fig. 5) were obvious in the higher dose nanocombination-treated group (Group Iab) compared to the infected non-treated control and the respective singly loaded PZQ monotherapy treated subgroups and in particular when given against the juvenile stage (Subgroup Ia 2). This denoted greater amelioration of schistosomiasis-induced pathology which results from immune-mediated granulomatous responses against Schistosoma eggs trapped in tissues. These are likely to cause serious local and systemic pathological effects associated with granuloma formation and fibrosis [53]. The significant reduction in hepatic granuloma size could be attributed to the distribution of PZQ $[13,54]$ and MFS $[55,56]$ to the liver of mice. Hepatic granulomas shrink progressively after PZQ treatment because of schistosome killing and reduction in the number of eggs trapped in the liver, reversing fibrogenesis [57].

In addition, adult male schistosomes recovered from $S$. mansoni-infected mice in the higher dose nanocombination subgroup (Iab) showed deformed suckers as well as extensive tegumental damage in the form of peeling of tubercles, disfigurement of the spines and appearance of subtegumental tissue (Fig. 3) supporting earlier observations for singly loaded PZQ LNCs [24] and MFS LNCs [16]. Suckers promote the attachment of schistosomes to blood vessels for the feeding process [1]. The tegument of Schistosoma also plays an important role in the uptake of nutrients and ions, excretion of metabolic end products 
as well as protection against the host immunological attack [58].

The obvious change observed in the therapeutic profile of nanoencapsulated PZQ induced by the addition of MFS in the higher nanocombination dose pointed to the benefit of combining drugs having different modes of action in appropriate dosing. This substantiated literature data for antischistosomal combinational therapies based exclusively on free drugs to date. Examples of PZQ combinations with enhanced efficacy in terms of reduced worm burden, or protection against pathological sequelae or multistage activity include PZQ-artesunate [59], PZQ-mefloquine [60, 61] and PZQ-edelfosine [62]. However, the fear of development of Plasmodium resistance to antimalarials and the relatively long treatment duration required for other combinations may hinder their applicability. This highlights the benefit of the current single oral dose nanoformulation.

Another great advantage of antischistosomal LNC formulations is their tegumental targeting ability observed earlier for MFS LNCs modified with oleic acid [16] and PZQ LNCs modified with oleic acid and a low concentration of MFS as formulation additives [24]. Such observations have been substantiated in the present study by high magnification SEM visualization of nano-objects deposited on the damaged tegument and the loose disfigured spines of adult S. mansoni worms (Fig. 3k, l). The size of these nano-objects was close to that of LNCs $(57.61 \pm 0.15 \mathrm{~nm})$. Noteworthy, no such nanostructures were observed for free unencapsulated MFS $[13,15]$ nor PZQ treatments [24]. Findings regarding these nanoobjects corroborate published data documenting possible intestinal translocation of LNCs across the intestinal wall $[29,63]$. Combined data emphasize the role of oral nanocarriers in mediating cellular drug delivery, providing new avenues for modified therapies [64].

A further objective of the study was to keep the doses of PZQ and MFS at the lowest level for effective therapy. Although the amounts of PZQ and MFS entrapped in the higher dose PZQ-MFS nanocombination were already reduced relative to the free drug forms [16, 43], an attempt was made to assess the effect of halving the dose of both drugs on the combined antischistosomal activity of a second lower fixed-dose nanocombination using the same procedures (Figs. 2, 5; Group II). Results indicated a statistically significant reduction in the antischistosomal activity of the lower nanocombination dose and its singly loaded control counterparts compared with the corresponding higher dose formulations.

Based on the results obtained, the antischistosomal activity of PZQ as the gold standard of antischistosomal therapy to date could be significantly enhanced by utilizing a multiple approach based on drug combination, drug repurposing and nanotechnology. Interaction of the nanocombination components resulted in the great promises offered by the higher dose PZQ-MFS nanocombination developed in the study. These include high efficacy against different developmental stages of experimental schistosomiasis mansoni infection at the reduced doses of $250 \mathrm{mg} / \mathrm{kg}$ of PZQ and $20 \mathrm{mg} / \mathrm{kg}$ of MFS. Efficacy enhancement was bestowed by the repurposing candidate, MFS, which also contributes to the structural stability and membrane activity of LNCs, probably facilitating tegumental targeting. Moreover, a statistically significant reduction in the size of hepatic granulomas, implied amelioration of a serious disease-associated liver pathology. Finally, a rationally selected nanocarrier (oleic-acid modified LNCs) showing controlled drug delivery and great benefits in oral administration contributed to efficacy enhancement by facilitating the intestinal translocation of the nanocombination and tegumental targeting.

\section{Conclusions}

The fixed-dose of $250 \mathrm{mg}$ PZQ-20 mg MFS $/ \mathrm{kg}$ nanocombination developed in the present study using LNCs offers advantages as a potential oral nanomedicine for single dose antischistosomal therapy with multistage activity and ability to ameliorate schistosomiasis-associated hepatic pathology. The study findings provide the first evidence on the benefits of integrating drug repurposing, combination therapy and nanotechnology as a novel multiple approach to improve the therapeutic profile of PZQ. Findings also verified the merits of LNCs as oral nanovectors and a carrier system for antischistosomal drug delivery, expanding their biomedical applications. From a clinical standpoint, utilization of the fixed-dose PZQ-MFS LNCs in MDA campaigns may offer great therapeutic potentials including a simpler administration process and radical cure allowed by multistage activity. Further studies are needed to document the safety of the developed nanocombination and explore its prophylactic activity, potential to hinder the onset of resistance to the differently acting drug components as well as cost effectiveness.

\footnotetext{
Abbreviations

EE\%: entrapment efficiency; Gl: gastrointestinal; H\&E: hematoxylin and eosin; LNCs: lipid nanocapsules; MDA: mass drug administration; MFS: miltefosine; PCS: photon correlation spectroscopy; Pdl: polydispersity index; p.i.: post-infection; PZQ: praziquantel; SEM: scanning electron microscopy; TEM: transmission electron microscopy; ZP: zeta potential.
}

Acknowledgements

The authors are grateful to Mrs Dawlat Ahmed for technical assistance with animal experimentation. 


\section{Authors' contributions}

MME and LEK conceived the study. MME, MZE and SAA designed the study. LKE and RME formulated and characterized the lipid nanocapsules. SAA and RME conducted the experiments. MME, MZE and SAA performed the SEM study. AA and SAA performed the histopathological study. MZE, LEK and SAA performed the data analysis. MME, MZE, LEK, AA, RME and SAA prepared the figures. MME, LEK and SAA wrote the manuscript. All authors read and approved the final manuscript.

\section{Funding}

The authors received no specific funding for this work.

\section{Availability of data and materials}

The data supporting the conclusions of this article are provided within the article.

\section{Ethics approval and consent to participate}

The study protocol was approved by the Ethics Committee of the Faculty of Medicine, Alexandria University, based on Egyptian regulations for animal experimentation (Protocol approval number: 0201101).

\section{Consent for publication}

Not applicable.

\section{Competing interests}

The authors declare that they have no competing interests.

\section{Author details}

${ }^{1}$ Department of Medical Parasitology, Faculty of Medicine, Alexandria University, Alexandria, Egypt. ${ }^{2}$ Department of Pharmaceutics, Faculty of Pharmacy, Alexandria University, Alexandria, Egypt. ${ }^{3}$ Department of Pathology, Faculty of Medicine, Alexandria University, Alexandria, Egypt.

Received: 8 February 2020 Accepted: 5 September 2020

Published online: 15 September 2020

\section{References}

1. LoVerde PT. Schistosomiasis. In: Toledo R, Fried B, editors. Digenetic trematodes. Cham: Springer International Publishing; 2019. p. 45-70.

2. Osakunor DN, Woolhouse ME, Mutapi F. Paediatric schistosomiasis: what we know and what we need to know. PLoS Negl Trop Dis. 2018;12:e0006144.

3. Wall KM, Kilembe W, Vwalika B, Dinh C, Livingston P, Lee YM, et al. Schistosomiasis is associated with incident HIV transmission and death in Zambia. PLoS Negl Trop Dis. 2018;12:e0006902.

4. Omar HH. Impact of chronic schistosomiasis and HBV/HCV co-infection on the liver: current perspectives. Hepat Med. 2019;11:131-6.

5. Woldegerima E, Bayih AG, Tegegne Y, Aemero M, Jejaw Zeleke A. Prevalence and reinfection rates of Schistosoma mansoni and praziquantel efficacy against the parasite among primary school children in Sanja town, Northwest Ethiopia. J Parasitol Res. 2019. https://doi. org/10.1155/2019/3697216.

6. Black CL, Steinauer ML, Mwinzi PN, Evan Secor W, Karanja DM, Colley DG Impact of intense, longitudinal retreatment with praziquantel on cure rates of schistosomiasis mansoni in a cohort of occupationally exposed adults in western Kenya. Trop Med Int Health. 2009;14:450-7.

7. Vale N, Gouveia MJ, Rinaldi G, Brindley PJ, Gartner F, Correia da Costa JM. Praziquantel for schistosomiasis: single-drug metabolism revisited, mode of action, and resistance. Antimicrob Agents Chemother. 2017:61:e02582-16.

8. da Silva VBR, Boucherle B, El-Methni J, Hoffmann B, da Silva AL, Fortune A, et al. Could we expect new praziquantel derivatives? A meta pharmacometrics/pharmacoinformatics analysis of all antischistosomal praziquantel derivatives found in the literature. SAR QSAR Environ Res. 2019;30:383-401.

9. Lyu H, Petukhov PA, Banta PR, Jadhav A, Lea W, Cheng Q, et al. Characterization of lead compounds targeting the selenoprotein thioredoxin glutathione reductase for treatment of schistosomiasis. ACS Infect Dis. 2020;6:393-405
10. Saccoccia F, Brindisi M, Gimmelli R, Relitti N, Guidi A, Saraswati AP, et al. Screening and phenotypical characterization of Schistosoma mansoni Histone deacetylase 8 (SmHDAC8) inhibitors as multistage antischistosomal agents. ACS Infect Dis. 2020;6:100-13.

11. Abou El Dahab MM, Shahat SM, Mahmoud SSM, Mahana NA. In vitro effect of curcumin on Schistosoma species viability, tegument ultrastructure and egg hatchability. Exp Parasitol. 2019;199:1-8.

12. Beshay EVN, Rady AA, Afifi AF, Mohamed AH. Schistosomicidal, antifibrotic and antioxidant effects of Cucurbita pepo L. seed oil and praziquantel combined treatment for Schistosoma mansoni infection in a mouse model. J Helminthol. 2019;93:286-94.

13. Eissa MM, El-Azzouni MZ, Amer El, Baddour NM. Miltefosine, a promising novel agent for schistosomiasis mansoni. Int J Parasitol. 2011;41:235-42.

14. Gouveia MJ, Brindley PJ, Gartner F, Costa J, Vale N. Drug repurposing for schistosomiasis: combinations of drugs or biomolecules. Pharmaceuticals. 2018;11:15.

15. Eissa MM, El Bardicy S, Tadros M. Bioactivity of miltefosine against aquatic stages of Schistosoma mansoni, Schistosoma haematobium and their snail hosts, supported by scanning electron microscopy. Parasit Vectors. 2011:4:73.

16. Eissa MM, El-Moslemany RM, Ramadan AA, Amer El, El-Azzouni MZ, El-Khordagui LK. Miltefosine lipid nanocapsules for single dose oral treatment of schistosomiasis mansoni: a preclinical study. PLoS ONE. 2015;10:e0141788.

17. El-Moslemany RM, Eissa MM, Ramadan AA, El-Khordagui LK, El-Azzouni MZ. Miltefosine lipid nanocapsules: intersection of drug repurposing and nanotechnology for single dose oral treatment of pre-patent schistosomiasis mansoni. Acta Trop. 2016;159:142-8.

18. Santos-Valle ABC, Souza GR, Paes CQ, Miyazaki T, Silva AH, Altube MJ, et al. Nanomedicine strategies for addressing major needs in neglected tropical diseases. Annu Rev Control. 2019;48:423-41.

19. Sun Y, Chen D, Pan Y, Qu W, Hao H, Wang X, et al. Nanoparticles for antiparasitic drug delivery. Drug Deliv. 2019;26:1206-21.

20. Labib El Gendy AEM, Mohammed FA, Abdel-Rahman SA, Shalaby TIA, Fathy GM, Mohammad SM, et al. Effect of nanoparticles on the therapeutic efficacy of praziquantel against Schistosoma mansoni infection in murine models. J Parasit Dis. 2019;43:416-25.

21. Radwan A, El-Lakkany NM, William S, El-Feky GS, Al-Shorbagy MY, Saleh $S$, et al. A novel praziquantel solid lipid nanoparticle formulation shows enhanced bioavailability and antischistosomal efficacy against murine $S$. mansoni infection. Parasites Vectors. 2019;12:304.

22. Zoghroban HS, El-Kowrany SI, Aboul Asaad IA, El Maghraby GM, El-Nouby KA, Abd Elazeem MA. Niosomes for enhanced activity of praziquantel against Schistosoma mansoni: in vivo and in vitro evaluation. Parasitol Res. 2019;118:219-34

23. Tawfeek GM, Baki MHA, Ibrahim AN, Mostafa MAH, Fathy MM, Diab $M$. Enhancement of the therapeutic efficacy of praziquantel in murine Schistosomiasis mansoni using silica nanocarrier. Parasitol Res. 2019:118:3519-33.

24. Amara RO, Ramadan AA, El-Moslemany RM, Eissa MM, El-Azzouni MZ, El-Khordagui LK. Praziquantel-lipid nanocapsules: an oral nanotherapeutic with potential Schistosoma mansoni tegumental targeting. Int J Nanomed. 2018:13:4493-505.

25. Carradori D, Labrak Y, Miron VE, Saulnier P, Eyer J, Preat V, et al. Retinoic acid-loaded NFL-lipid nanocapsules promote oligodendrogenesis in focal white matter lesion. Biomaterials. 2020;230:119653.

26. Fabbri J, Espinosa JP, Pensel PE, Medici SK, Gamboa GU, Benoit JP, et al. Do albendazole-loaded lipid nanocapsules enhance the bioavailability of albendazole in the brain of healthy mice? Acta Trop. 2020;201:105215.

27. Huynh NT, Passirani C, Saulnier P, Benoit JP. Lipid nanocapsules: a new platform for nanomedicine. Int J Pharm. 2009;379:201-9.

28. Ullio Gamboa GV, Pensel PE, Elissondo MC, Sanchez Bruni SF, Benoit JP, Palma SD, et al. Albendazole-lipid nanocapsules: optimization, characterization and chemoprophylactic efficacy in mice infected with Echinococcus granulosus. Exp Parasitol. 2019;198:79-86.

29. Varshosaz J, Taymouri S, Jahanian-Najafabadi A, Alizadeh A. Efavirenz oral delivery via lipid nanocapsules: formulation, optimisation, and ex-vivo gut permeation study. IET Nanobiotechnol. 2018;12:795-806.

30. Derakhshani A, Rezaei Z, Safarpour H, Sabri M, Mir A, Sanati MA, et al. Overcoming trastuzumab resistance in HER2-positive breast cancer using combination therapy. J Cell Physiol. 2020;235:3142-56. 
31. Kawazoe A, Shitara K. Trifluridine/tipiracil for the treatment of metastatic gastric cancer. Expert Rev Gastroenterol Hepatol. 2020;14:65-70.

32. Coates ARM, Hu Y, Holt J, Yeh P. Antibiotic combination therapy against resistant bacterial infections: synergy, rejuvenation and resistance reduction. Expert Rev Anti Infect Ther. 2020;18:5-15.

33. Hu CM, Aryal S, Zhang L. Nanoparticle-assisted combination therapies for effective cancer treatment. Ther Deliv. 2010;1:323-34.

34. Al-Attar T, Madihally SV. Recent advances in the combination delivery of drug for leukemia and other cancers. Expert Opin Drug Deliv. 2020;17:213-23.

35. Zhao M, van Straten D, Broekman MLD, Preat V, Schiffelers RM. Nanocarrier-based drug combination therapy for glioblastoma. Theranostics. 2020;10:1355-72.

36. Walvekar P, Gannimani R, Govender T. Combination drug therapy via nanocarriers against infectious diseases. Eur J Pharm Sci. 2019;127:121-41.

37. Pawar S, Shende P. 2(2) factorial design-based biocompatible microneedle arrays containing artemether co-loaded with lumefantrine nanoparticles for transepidermal delivery. Biomed Microdevices. 2020;22:19

38. Shakeel K, Raisuddin S, Ali S, Imam SS, Rahman MA, Jain GK, Ahmad FJ. Development and in vitro/in vivo evaluation of artemether and lumefantrine co-loaded nanoliposomes for parenteral delivery. J Liposome Res. 2019;29:35-43. https://doi.org/10.1080/08982104.2017.1410173.

39. Tomiotto-Pellissier F, Miranda-Sapla MM, Machado LF, Bortoleti B, Sahd CS, Chagas AF, et al. Nanotechnology as a potential therapeutic alternative for schistosomiasis. Acta Trop. 2017;174:64-71.

40. Lombardo FC, Pasche V, Panic G, Endriss Y, Keiser J. Life cycle maintenance and drug-sensitivity assays for early drug discovery in Schistosoma mansoni. Nat Protoc. 2019;14:461-81.

41. Smithers SR, Terry RJ. The infection of laboratory hosts with cercariae of Schistosoma mansoni and the recovery of the adult worms. Parasitology. 1965;55:695-700.

42. Jeffree CE, Read ND. Ambient-and low-temperature scanning electron microscopy. In: Hall JL, Hawes C, editors. Electron microscopy of plant cells. New York: Academic Press; 1991. p. 313-413.

43. Le L, Molehin AJ, Nash S, Sennoune SR, Ahmad G, Torben W, et al. Schistosoma egg-induced liver pathology resolution by Sm-p80-based schistosomiasis vaccine in baboons. Pathology. 2018;50:442-9.

44. Chan JD, Zarowiecki M, Marchant JS. Ca2+ channels and praziquantel: a view from the free world. Parasitol Int. 2013;62:619-28.

45. Park SK, Gunaratne GS, Chulkov EG, Moehring F, McCusker P, Dosa PI, et al. The anthelmintic drug praziquantel activates a schistosome transient receptor potential channel. J Biol Chem. 2019;294:18873-80.

46. Monge-Maillo B, Lopez-Velez R. Miltefosine for visceral and cutaneous leishmaniasis: drug characteristics and evidence-based treatment recommendations. Clin Infect Dis. 2015;60:1398-404

47. Fernandes KS, de Souza PE, Dorta ML, Alonso A. The cytotoxic activity of miltefosine against Leishmania and macrophages is associated with dynamic changes in plasma membrane proteins. Biochim Biophys Acta Biomembr. 2017;1859:1-9.

48. Moreira RA, Mendanha SA, Fernandes KS, Matos GG, Alonso L, Dorta $\mathrm{ML}$, et al. Miltefosine increases lipid and protein dynamics in Leishmania amazonensis membranes at concentrations similar to those needed for cytotoxicity activity. Antimicrob Agents Chemother. 2014;58:3021-8.

49. El Ridi R, Tallima H. Equilibrium in lung schistosomula sphingomyelin breakdown and biosynthesis allows very small molecules, but not antibody, to access proteins at the host-parasite interface. J Parasitol. 2006:92:730-7.
50. Bertao HG, da Silva RA, Padilha RJ, de Azevedo Albuquerque MC, RadisBaptista G. Ultrastructural analysis of miltefosine-induced surface membrane damage in adult Schistosoma mansoni BH strain worms. Parasitol Res. 2012;110:2465-73.

51. Xiao SH, Catto BA, Webster LT Jr. Effects of praziquantel on different developmental stages of Schistosoma mansoni in vitro and in vivo. J Infect Dis. 1985;151:1130-7.

52. Aragon AD, Imani RA, Blackburn VR, Cupit PM, Melman SD, Goronga T, et al. Towards an understanding of the mechanism of action of praziquantel. Mol Biochem Parasitol. 2009;164:57-65.

53. Masi B, Perles-Barbacaru TA, Bernard M, Viola A. Clinical and preclinical imaging of hepatosplenic schistosomiasis. Trends Parasitol. 2020;36:206-26.

54. Li-zhi W, Xin-Sheng Z, Jiang-shan D, Yi W, Bao-an Y. Transdermal praziquantel administration attenuates hepatic granulomatosis in mice infected with Schistosoma japonicum. Parasitol Res. 2015;114:1417-24.

55. Breiser A, Kim DJ, Fleer EA, Damenz W, Drube A, Berger M, et al. Distribution and metabolism of hexadecylphosphocholine in mice. Lipids. 1987;22:925-6

56. Jimenez-Anton MD, Garcia-Calvo E, Gutierrez C, Escribano MD, Kayali N, Luque-Garcia $J$, et al. Pharmacokinetics and disposition of miltefosine in healthy mice and hamsters experimentally infected with Leishmania infantum. Eur J Pharm Sci. 2018;121:281-6.

57. Morsy G. Parasitological and histo-pathological studies on schistosomiasis mansoni infected mice and treated with praziquatel and/or oltipraz. J Egypt Soc Parasitol. 2009;39:687-701.

58. Xiao S, Binggui S, Chollet J, Tanner M. Tegumental changes in 21-day-old Schistosoma mansoni harboured in mice treated with artemether. Acta Trop. 2000;75:341-8.

59. Hegazy LAM, Motiam MHA, Abd El-Aal NF, Ibrahim SM, Mohamed HK. Evaluation of artesunate and praziquantel combination therapy in murine schistosomiasis mansoni. Iran J Parasitol. 2018;13:193-203.

60. Keiser J, ManneckT, Vargas M. Interactions of mefloquine with praziquantel in the Schistosoma mansoni mouse model and in vitro. J Antimicrob Chemother. 2011;66:1791-7.

61. Manneck T, Braissant O, Ellis W, Keiser J. Schistosoma mansoni: antischistosomal activity of the four optical isomers and the two racemates of mefloquine on schistosomula and adult worms in vitro and in vivo. Exp Parasitol. 2011;127:260-9.

62. Yepes E, Varela MR, Lopez-Aban J, Rojas-Caraballo J, Muro A, Mollinedo F. Inhibition of granulomatous inflammation and prophylactic treatment of schistosomiasis with a combination of edelfosine and praziquantel. PLoS Negl Trop Dis. 2015;9:e0003893.

63. Roger E, Gimel JC, Bensley C, Klymchenko AS, Benoit JP. Lipid nanocapsules maintain full integrity after crossing a human intestinal epithelium model. J Control Release. 2017;253:11-8.

64. Ghosh S, Ghosh S, Sil PC. Role of nanostructures in improvising oral medicine. Toxicol Rep. 2019;6:358-68.

\section{Publisher's Note}

Springer Nature remains neutral with regard to jurisdictional claims in published maps and institutional affiliations.

Ready to submit your research? Choose BMC and benefit from:

- fast, convenient online submission

- thorough peer review by experienced researchers in your field

- rapid publication on acceptance

- support for research data, including large and complex data types

- gold Open Access which fosters wider collaboration and increased citations

- maximum visibility for your research: over 100M website views per year

At BMC, research is always in progress.

Learn more biomedcentral.com/submissions 